\title{
CONTRIBUTION OF HIPPOCAMPAL PLACE CELL ACTIVITY TO LEARNING AND FORMATION OF GOAL-DIRECTED NAVIGATION IN RATS
}

\author{
T. KOBAYASHI, ${ }^{a}$ A. H. TRAN, ${ }^{a}$ H. NISHIJO,,,$c$ T. ONO \\ AND G. MATSUMOTO ${ }^{a *}$ \\ ${ }^{a}$ Brain-Operative Expression Team, Brainway Group, Brain Science \\ Institute, RIKEN (The Institute of Physical and Chemical Research), \\ Hirosawa, Wako, Saitama 351-0198, Japan \\ ${ }^{b}$ Department of Physiology, Faculty of Medicine, Toyama Medical and \\ Pharmaceutical University, Sugitani, Toyama 930-0194, Japan \\ ${ }^{\circ}$ CREST, JST, Chiyoda-ku, Tokyo 101-0032, Japan
}

\begin{abstract}
Although extensive behavioral studies have demonstrated that hippocampal lesions impair navigation toward specific places, the role of hippocampal neuronal activity in the development of efficient navigation during place learning remains unknown. The aim of the present study was to investigate how hippocampal neuronal activity changes as rats learn to navigate efficiently to acquire rewards in an open field. Rats were pre-trained in a random reward task where intracranial self-stimulation rewards were provided at random locations. Then, the rats were trained in a novel place task where they were rewarded at two specific locations as they repeatedly shuttled between them. Hippocampal neuronal activity was recorded during the course of learning of the place task. The rats learned reward sites within several sessions, and gradually developed efficient navigation strategies throughout the learning sessions. Some hippocampal neurons gradually changed spatial firing as the learning proceeded, and discharged robustly near the reward sites when efficient navigation was established. Over the learning sessions, the neuronal activity was highly correlated to formation of efficient shuttling trajectories between the reward sites. At the end of the experiment, spatial firing patterns of the hippocampal neurons were re-examined in the random reward task. The specific spatial firing patterns of the neurons were preserved if the rats navigated, as if they expected to find rewards at the previously valid locations. However, those specific spatial firing patterns were not observed in rats pursuing random trajectories.

These results suggest that hippocampal neurons have a crucial role in formation of an efficient navigation. () 2003 IBRO. Published by Elsevier Science Ltd. All rights reserved.
\end{abstract}

Key words: hippocampal formation, unit recording, plasticity, place learning.

Navigation toward a specific place in the environment is crucial for survival for animals as well as humans. Behavioral experiments in rats have shown that the hippocampal-

*Corresponding author. Tel: +81-48-467-9635; fax: +81-48-4679643.

E-mail address: gen@brainway.riken.go.jp (G. Matsumoto).

Abbreviations: HF, hippocampal formation; ICSS, intracranial self-stimulation; RRPST, random-reward place-search task; PLT, place-learning task. formation (HF) damage severely impairs navigation and place learning (O'Keefe and Nadel, 1978; Olton et al., 1979; Jarrard, 1993). Neuropsychological studies showed patients with damage in the medial temporal lobe including the HF and the parahippocampal gyrus had deficits in topographical orientation (Maguire et al., 1996; Abrahams et al., 1997). Recent neuroimaging studies have also shown that activity in these regions increases during navigation in a virtual reality space (Maguire et al., 1998) and recalling actual routes (Maguire et al., 1997; Mellet et al., 2000). All of these studies indicated that the HF has a crucial role for spatial navigation, not just for spatial recognition.

The discovery of the place cell in the HF (O'Keefe and Dostrovsky, 1971) provided a neurophysiological approach to examine the role of the HF in spatial functions, and subsequent studies revealed characteristics of spatial representation in the HF (Muller and Kubie, 1987; O'Keefe and Speakman, 1987; Eichenbaum et al., 1989; McNaughton et al., 1996). The place cell of the rodent HF is characterized by location-specific firing (O'Keefe and Conway, 1978; Eichenbaum et al, 1987; McHugh et al, 1996; Skaggs and McNaughton, 1998), and comparable HF neuron responses were also reported in monkeys (Ono et al., 1991, 1993; Nishijo et al., 1997; Matsumura et al., 1999). Place-cell activity is controlled by a salient cue or multiple spatial cues (e.g., O'Keefe and Conway, 1978; Muller and Kubie, 1987; O'Keefe and Burgess, 1996), and remains stable over a long period (Muller et al., 1987; Thompson and Best, 1990). These studies suggest that place-cell activity reflects a spatial representation of an environment.

However, alterations of behavioral as well as spatial variables induce plastic changes in place-cell firing. Placecell activity changes in an experience-dependent manner (Bostock et al., 1991; Wilson and McNaughton, 1993; Mehta et al., 1997, 2000). Alteration of reward-place associations also induces changes in locations of place fields (Breese et al., 1989; Kobayashi et al., 1997). Furthermore, Markus et al. (1995) indicate that the place cell flexibly changes its spatial firing as rats learn to navigate to specific locations. However, it remains unclear how spatial firing of place cells contributes to development of navigation directed to specific goals during place learning.

For the understanding of functions of the HF, it is important to investigate the actual role of place cells in spatial navigation (Bures et al., 1997a, 1997b; Zinyuk et al., 2000; Lenck-Santini et al., 2001). A purpose of the present study is to elucidate relationships between activity of HF neurons and goal-directed behaviors acquired dur- 
ing learning of a novel place task. Some of these data have been reported in abstract form (Kobayashi et al., 1991; Nishijo et al., 1999).

\section{EXPERIMENTAL PROCEDURES}

\section{Subjects}

Thirteen male albino Wistar rats (Sankyo Lab Service, Hamamatsu, Japan) were used. The rats weighed $270-320 \mathrm{~g}$ at the time of surgery, and were individually housed in a room with constant temperature and had food and water freely available. Efforts were made to minimize the number of animals used and their suffering. All rats were treated in strict compliance with the United States Public Health Service Policy on Human Care and Use of Laboratory Animals and the National Institutes of Health Guide for the Care and Use of Laboratory Animals, and the Guideline for the Care and Use of Laboratory Animals in Toyama Medical and Pharmaceutical University.

\section{Surgery}

Each rat was anesthetized with pentobarbital sodium $(40 \mathrm{mg} / \mathrm{kg}$, i.p.) and implanted bilaterally with monopolar stimulating electrodes (enamel insulated, 200- $\mu \mathrm{m}$ diameter, stainless steel) aimed at the medial forebrain bundle at the level of the lateral hypothalamus $(4.3 \mathrm{~mm}$ caudal from the bregma, $1.6 \mathrm{~mm}$ lateral from the midline, and $8.8-9.0 \mathrm{~mm}$ ventral from the skull surface), and a recording electrode assembly above the CA1 layer of the dorsal HF (3.6 mm caudal from the bregma, $3.0 \mathrm{~mm}$ lateral from the midline and $2.8 \mathrm{~mm}$ below the skull surface) according to the atlas of Paxinos and Watson (1986). This coordination allowed us to record neuronal activity not only in the CA1 but also CA3 layers. The recording electrode assembly consisted of a bundle of four or eight wires (Formvar insulated, $20-\mu \mathrm{m}$ diameter, nichrome) encased in a stainless steel cannula (25 gauge), a small platform, and two screws. Stimulating electrodes and a recording electrode assembly were attached to the skull with dental acrylic and stainless steel screws, one of which also served as the indifferent electrode for stimulation.

\section{Apparatus}

Spatial behavior was investigated in a 150-cm-diameter circular open field with a $45-\mathrm{cm}$-high wall, painted black on the inside. The open field was enclosed by a black curtain $(180 \mathrm{~cm}$ in diameter and $200 \mathrm{~cm}$ high). The ceiling of the enclosure contained four small speakers mounted near the circumference, spaced $90^{\circ}$ apart, four light bulbs individually mounted near the inner edge of each speaker, and a video camera (Behavioral Tracking Analyzer, BTA-2A, Muromachi Kikai, Tokyo, Japan) at the center. Usually a light bulb was lit at the three o'clock position, and a speaker continuously emitted white noise at the nine o'clock position. A small light bulb was mounted on the head of the rat. The video camera converted a real video image signal to a binary signal, and tracked the horizontal (two-dimensional) motion of the small bulb. A laboratory microcomputer (PC-9801, NEC, Tokyo, Japan) received the $X$ and $Y$ coordinates of the position of the head through an RS-232C serial port at 20 frames/s. A program delimited circular areas (reward sites) in the open field, and triggered the delivery of intracranial self-stimulation (ICSS) when the rat entered the reward site. The experimenter monitored the locations of the reward site and the rat on a display screen, but no distinctive cues marked the reward site for the rat.

\section{Behavioral Procedures}

Self-stimulation screening After at least 1 week recovery from surgery, the rats were screened to self-stimulate in an oper- ant chamber $(30 \times 30 \times 33 \mathrm{~cm})$ equipped with a lever on one wall. Each lever press triggered the delivery of a 0.5 -s train of $0.3-\mathrm{ms}$ negative square-wave pulses at $100 \mathrm{~Hz}$. The current intensity for ICSS was determined to produce 40-70 lever presses/min in the operant chamber. A stimulating electrode, which produced stable lever pressing at a low current intensity was selected for each rat. The rats were trained to self-stimulate in daily 30-60-min sessions for 5-10 days until stable lever pressing was achieved. The current intensity, which was determined in this period for each rat, ranged from 100 to $300 \mu \mathrm{A}$, and was used throughout the following place tasks in the open field.

Task training and testing protocol in open field. The rats were pre-trained to perform the following spatial behavior in the open field. ICSS was delivered when the cumulative distance traveled by the rat reached a given distance. The initial distance was $80 \mathrm{~cm}$, and this was increased progressively to 120 or 160 $\mathrm{cm}$. The rats were usually trained for about $1 \mathrm{~h} /$ day for 2-4 days until they learned to travel the open field continuously. They were then trained in the random-reward place-search task (RRPST), In this protocol a reward site $(72-\mathrm{cm}$ diameter) was delimited; its center was chosen at random within a square circumscribed around the open field (Fig. 1Aa). The rat was rewarded with ICSS when it entered the reward site, which was then made inactive. After a 5-s interval, the reward site was moved to a different location and reactivated.

After the rats obtained at least 30 rewards in each session of successive three sessions of the RRPST, the recording electrode assembly was advanced at about $20-80 \mu \mathrm{m}$ per day in $20-\mu \mathrm{m}$ steps. The electrodes in each rat were checked while the rat performed the RRPST. A purpose of the present study was to elucidate activity of HF neurons during new learning of goaldirected behaviors. Then, each animal could only be examined once. If a complex spike cell was found, and stable recording was obtained, data for this rat were collected in 1 day as follows. Initially data were recorded in a several sessions of the RRPST, and then five to 10 sessions of place learning task (PLT) were run. In the PLT, two 40-cm-diameter reward sites were located diametrically opposite to one another in the open field at separation of $70 \mathrm{~cm}$ (Fig. 1Ab). In each session the first entrance into the north reward site was always rewarded. Then, the rat was rewarded in both reward sites on the condition that it had previously visited the other one and remained within the reward site for $1 \mathrm{~s}$. This delay was intended to ensure that the rat identified the reward site as such, and was not rewarded for simply arriving at the reward site by automatic locomotion.

At the start of a session, the small electric bulb on the head of the rat was turned on, and an ICSS train was delivered to prime the rat. Each session was terminated after 50 rewards had been delivered or 10 min had elapsed, whichever occurred first.

\section{Recording procedures and data acquisition}

Neuronal activity was passed through a high-input impedance preamplifier made of a dual-channel field-effect transistor (2SK389, Toshiba Electric, Tokyo, Japan) or a FET-input op-amp (AD824, Analog Devices, Norwood, MA, USA), amplified by a main amplifier, and then fed into time amplitude window discriminators (BAK Electronics, Germantown, MD, USA) to convert to standardized pulses. During ICSS, neuronal activity was isolated from the stimulus artifact by the time-window discriminator. Differential recording with these electrodes and recording electrode assembly has been shown to yield stable, single neuronal activity (Sasaki et al., 1983; McNaughton et al., 1989). The activity of a neuron was recorded as a single neuronal activity if it had a peak amplitude at least 2.0 times greater than noise, and the amplitude, duration (spike width), and waveform were confirmed to be constant by visual examination on a storage oscilloscope (Fig. 1B). In the cases in which one electrode had multiple neurons, single 
A

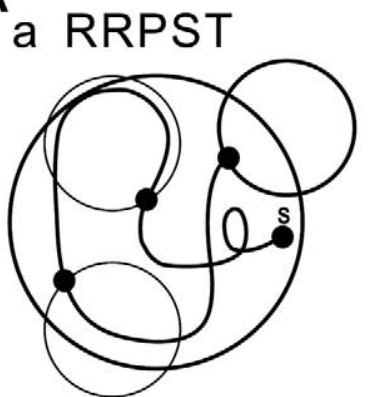

b PLT

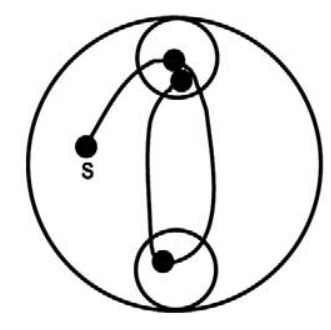

B

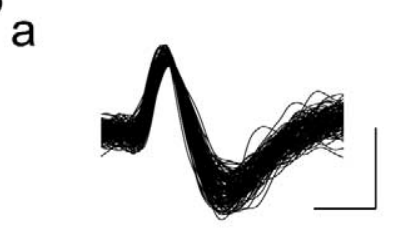

b

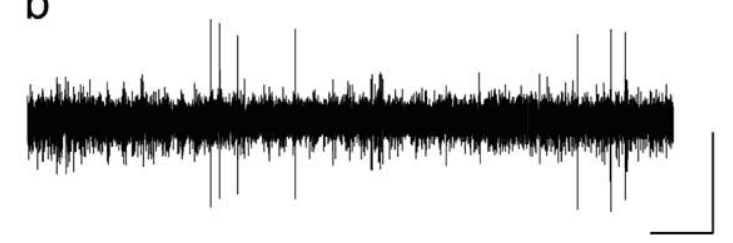

Fig. 1. Behavioral tasks and examples of place cell activity. (Aa) Random reward place search task (RRPST). A computer program delimited a circular reward site (small thick-line circle) at some randomly selected coordinate. The rat was rewarded with intracranial self-stimulation when it entered the reward site, which was then made inactive (changed to thin-line circle). After a 5-s interval, the reward site was moved to a different location and reactivated. (Ab) Placelearning task (PLT). The rat received rewards in two target areas (thick-line circles) when it returned to one reward site after a visit to the other reward site. There was a 1-s delay between arrival and reward delivery. S, location of rat at start of session. Large dots, locations of reward delivery. $(\mathrm{Ba})$ Superimposed waveforms for the activity of a hippocampal place cell. Vertical and horizontal bars, calibration for amplitude $(0.1 \mathrm{mV})$ and time $(0.2 \mathrm{~ms})$, respectively. $(\mathrm{Bb})$ An example of neuronal activity of the place cell. Vertical and horizontal bars, calibration for amplitude $(0.1 \mathrm{mV})$ and time $(0.5 \mathrm{~s})$, respectively.

neuronal activities were identified and recorded by means of separation with the time amplitude window discriminator (Sakurai, 1996). Data sets were discarded if the neurons were judged to be poorly isolated. The microcomputer summed the spikes (standardized pulses) over 50-ms intervals and combined these data with the $X$ and $Y$ coordinates of the head of the rat to construct $a$ distribution map of the mean firing rate as a function of the position of the rat.

\section{Data representation and analysis}

To represent the firing rate distribution in the open field, firing-rate maps consisting of $4.5 \times 4.5-\mathrm{cm}$ pixels were constructed. Dividing the total number of spikes by the cumulative dwell time in each pixel for the entire session yielded the firing-rate map. Pixels where the rat visited but a given neuron did not fire were represented by gray pixels. Pixels with greater than zero firing rate were sorted into ascending order and divided into five color-scale categories. The values used as boundaries of the five color categories increased at a step of half of mean firing rate, and pixels with the highest firing categories (greater than twice the mean firing rate) were shown as red pixels. To permit comparisons among firing-rate distributions across several sessions for a given cell, the rate categories determined for the first map were retained for the subsequent maps.

To estimate similarity of trajectories and firing-rate distributions in each session to those in the final session of the PLT, pixel-to-pixel correlations (Sharp et al., 1990) were calculated using the dwell-time map and firing-rate map (see above), respectively. For the dwell-time map, a smoothing algorithm was applied in that the value of a given pixel, the values of the four adjacent pixels that share an edge with it, and the values of the four adjacent pixels that share only one corner with the center were divided by four, eight, 16 , respectively, and then summed to generate the map to calculate trajectory similarity. The rat gradually spent more and more time near and within the reward site during learning the PLT, which falsely increased pixel-to-pixel correlation for trajectory similarity. Therefore, to accurately estimate trajectory similarity, the pixels near and within the reward site were excluded from the calculation. To estimate the similarity of firing-rate distributions, firing-rate maps consisting of $9.0 \times 9.0-\mathrm{cm}$ pixels were generated. The same smoothing algorithm used for the trajectory similarity analysis was applied to these maps. Then, pixel-to-pixel correlation was calculated using only common pixels between the final session of the PLT and each session.

Place fields were delineated by a process used previously (Muller et al., 1987; Breese et al., 1989; Kobayashi et al., 1997). Clusters of $4.5 \times 4.5-\mathrm{cm}$ pixels with firing rates exceeding twice the mean firing rate were identified. All pixels that did not satisfy this criterion were eliminated. A place field could be continued through any edge shared by two pixels meeting the criterion, but not through corners. If one or more neighboring pixels satisfied the criterion, the field was expanded to include the pixel(s). Each added pixel was then tested for the presence of a neighboring pixel that met the criterion. When no neighboring pixel satisfied the criterion, the limit of the field was identified. The minimum size for a place field was set at nine pixels. Boundaries of a place field were established by constructing a rectangle that had one diagonal connecting the minimum $X$ and $Y$ coordinates with the maximum $X$ and $Y$ coordinates.

\section{Histology}

After the recording electrodes were advanced below the HF, the locations of the recording and stimulating electrodes were identified histologically. The rats were deeply anesthetized with pentobarbital sodium (50 mg/kg, i.p.), and the stimulating site was marked by an iron deposit, created by passing a $20-\mu \mathrm{A}$ positive current through the stimulating electrode for $30 \mathrm{~s}$. The recording sites were marked by an electrolytic lesion, created by passing a $20-\mu \mathrm{A}$ negative current through the recording electrodes for $30 \mathrm{~s}$. For marking of the stimulating and recording sites, the stainless steel screws to attach the recording electrode assembly to the skull were used as the indifferent electrodes. Each rat was then perfused through the heart with $50 \mathrm{ml}$ of $0.9 \%$ saline containing heparin followed by $200 \mathrm{ml}$ of $10 \%$ buffered formalin containing $2 \%$ potassium ferrocyanide. The brain was removed and fixed in formalin for at least $48 \mathrm{~h}$. Sections of $75 \mu \mathrm{m}$ were cut on a freezing microtome and were then stained with Cresyl Violet.

\section{RESULTS}

\section{Behavioral performance}

Figure 2 summarizes the results of behavioral performance of 13 rats during learning of the PLT. The rats acquired 19.54 \pm 4.68 (mean \pm S.E.M.) rewards in the first session, and $46.54 \pm 3.14$ rewards in the last session of the 


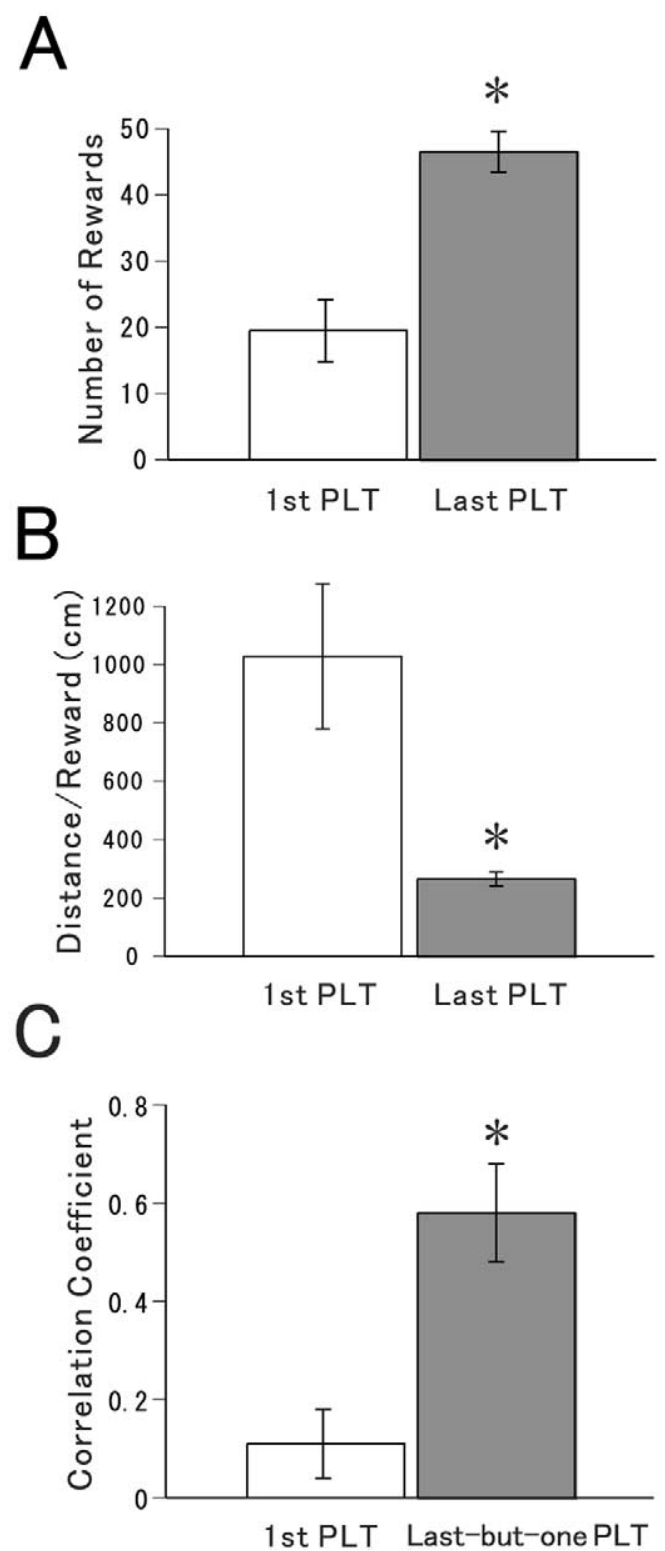

Fig. 2. Behavioral performance for learning the place-learning task PLT. (A) mean number of rewards obtained in the first and last session of the PLT. (B) Efficiency of obtaining rewards in the first and last session of the PLT. Efficiency was calculated as dividing traveled distance by total number of rewards in each session. (C) Trajectory similarity. Trajectory similarity was calculated as correlation coefficients between the first and last sessions and between the last-butone and last sessions of the PLT (mean \pm S.E.M.; ${ }^{*} P<0.01$; $t$-test, $n=13)$.

PLT (Fig. 2A). There was a significant improvement in the number of rewards between the first and last sessions of the PLT (Student's $t$-test, $P<0.01$ ). The efficiency of acquiring rewards was quantified by dividing the traveled distance by the total number of rewards that the rats acquired in each session (Fig. 2B). The rats traveled $1028.4 \pm 249.2 \mathrm{~cm}$ per reward in the first session, and $265.9 \pm 24.4 \mathrm{~cm}$ per reward in the last session of the PLT. There was a significant improvement in the traveled distance per reward between the first and last sessions of the
PLT (Student's $t$-test, $P<0.01$ ), indicating that the rats acquired rewards more efficiently in the last session. Trajectories of the rats also greatly changed across the sessions of the PLT (Fig. 2C). The trajectory similarity between the first and last sessions of the PLT was low $\left(r_{\text {trajectory }}=0.11 \pm 0.07\right)$, whereas the trajectory in the session immediately before the last session more resembled that in the last session $\left(r_{\text {trajectory }}=0.58 \pm 0.10\right)$ (Student's $t$-test, $P<0.01)$. These results indicate that the rats learned the PLT well, and, as learning proceeded, the pattern of trajectories also changed for efficient reward acquisition.

\section{Changes in firing rate distributions during learning the PLT}

The activity of 14 complex-spike cells was recorded from 13 rats. Each complex-spike cell was initially recorded in a several sessions of the RRPST and the following five to 10 sessions of the PLT. Data for each rat were collected in 1 day. To estimate whether or not spatial firing patterns changed after learning the PLT and to characterize the neurons, we used correlation coefficients $\left(r_{\text {rate }}\right)$ between firing-rate distributions in the last session of the RRPST before PLT learning and the last session of the PLT. Neurons which had high $r_{\text {rate }}$ values $\left(r_{\text {rate }} \geq 0.4\right)$ were considered to have maintained the same spatial firing patterns, whereas neurons which had low $r_{\text {rate }}$ values $\left(r_{\text {rate }}<0.4\right)$ were defined as having changed spatial firing patterns after learning the PLT. Four neurons (29\%) belonged to the first group (mean $r_{\text {rate }}=0.48 \pm 0.06$ ), and 10 neurons $(71 \%)$ belonged to the second group (mean $r_{\text {rate }}=$ $0.03 \pm 0.05)$.

Figure 3 shows an example of trajectories and spatial firing patterns of a CA1 neuron which maintained the spatial firing patterns in the RRPST after the PLT was learned. Activity of the neuron increased when the rat passed through the location around one to two o'clock in the RRPST before the PLT (Fig. 3A). This spatial firing pattern was preserved over subsequent learning sessions (Fig. 3B-E). The spatial firing pattern in the last session of the PLT learning (Fig. 3E) resembled that in the RRPST before learning $\left(r_{\text {rate }}=0.65\right)$. Furthermore, the spatial firing pattern in the RRPST after learning the PLT (Fig. 3F) was also similar to that in the last session of PLT learning $\left(r_{\text {rate }}=0.61\right)$.

Figure 4 shows an example of trajectories and spatial firing patterns of a CA1 neuron which changed spatial firing patterns in the RRPST after PLT learning. Spatial firing patterns were diffuse in the RRPST before learning the PLT and in the initial three sessions of the PLT (Fig. $4 \mathrm{~A}-\mathrm{C})$. In the fifth session of the PLT, the rat was clearly orienting toward two reward sites, and activity increased when the rat was near or within the reward site at 12 o'clock location (Fig. 4D). In the final session of the PLT (Fig. 4E), spatial firing near the reward site at 12 o'clock location was more evident, and activity of this neuron increased before reward delivery and decreased after the delivery. In the RRPST after learning the PLT, both the goal-directed shuttling behavior and spatial firing pattern acquired in the PLT learning session were preserved (Fig. 


\section{A RRPST before PLT learning D PLT 7th Session}
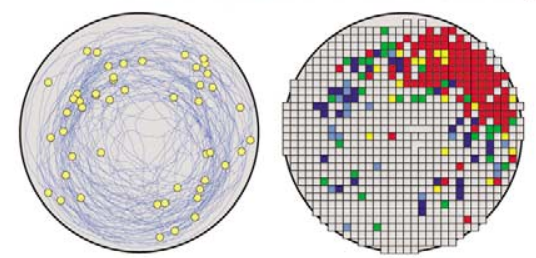

B PLT 1st Session
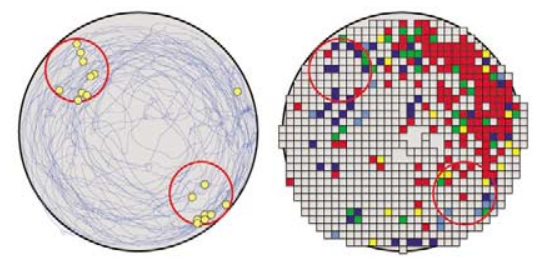

\section{PLT 4th Session}
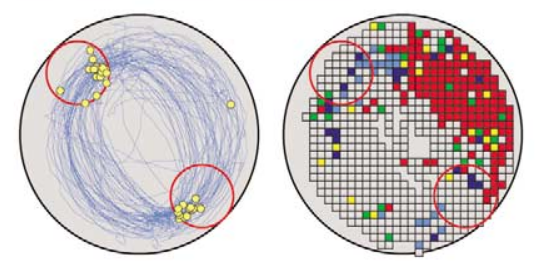
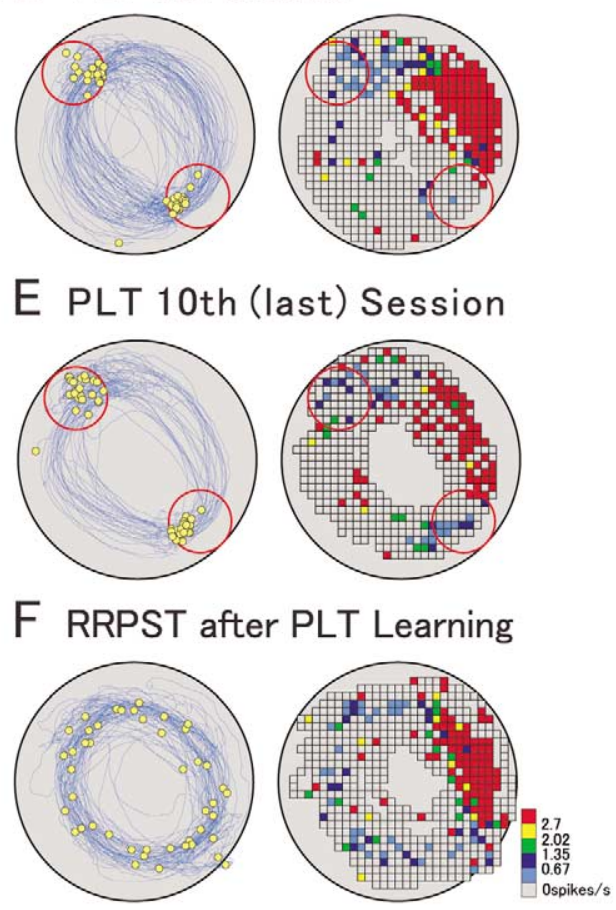

Fig. 3. An example of trajectories and firing-rate distribution of a hippocampal-formation neuron whose spatial firing pattern did not change during the learning of the place-learning task (PLT). (A) Random-reward place-search task (RRPST) before learning. (B) First session of the PLT. (C) Fourth session of the PLT. (D) Seventh session of the PLT. (E) Tenth session of the PLT. (F) RRPST after the learning of the PLT. This neuron had a place field at the 1 to 2 o'clock location along the cylinder wall in the RRPST before the PLT was learned (A). The sense of rotation of the rat was indefinite in the first PLT session, but became counterclockwise from the fourth session. This spatial firing pattern was maintained over subsequent learning sessions (B-E) and in the RRPST after learning (F). Blue lines, trajectories of rat. Large yellow dots, locations of reward delivery. Red circles, locations of reward sites. Pixels with greater than zero firing rate were divided into five color-scale categories, and the values used as boundaries of these categories are shown in calibrations. Pixels where the rat visited and the cell never fired are represented by gray pixels. A-F are data from the same neuron.

4F). The trajectory in the RRPST after the PLT was highly correlated to that in the last session of the PLT (see below in detail). Figure 5 shows changes in number of rewards and efficiency of acquiring rewards (A), and the trajectory and firing rate distribution similarity of the same neuron (B) as a function of session. The rat was already able to acquire over 30 rewards in the initial three sessions, and earned 50 rewards in the last two sessions of the PLT. On the other hand, traveled distance per reward linearly decreased as learning proceeded (Fig. 5A). Both the trajectory similarity $\left(r_{\text {trajectory }}\right)$ and the firing rate similarity $\left(r_{\text {rate }}\right)$ increased as learning proceeded (Fig. 5B), and the values of these similarities were highly correlated $(r=0.84$, $P<0.05)$. It should be noted that high values of $r_{\text {trajectory }}$ as well as $r_{\text {rate }}$ were preserved in the RRPST after sessions of the PLT, indicating that both behavioral and spatial firing patterns in this session resembled those in the last session of the PLT learning as shown in Fig. 4E and F.

Another example of PLT learning-related changes in trajectories and spatial firing patterns of a CA3 neuron is shown in Fig. 6. The spatial firing pattern was diffuse in the RRPST before PLT learning (Fig. 6A). In the initial sessions of the PLT, spatial firing patterns were indistinct or unstable (Fig. 6B and C). However, in the last two sessions of the PLT, where the rat clearly directed its course toward the goal locations, the neuronal activity increased when the rat was near or within the reward site at the 12 o'clock location (Fig. 6D and E). In contrast with the neuron shown in Fig. 4, these behavioral and spatial firing patterns were not preserved in a subsequent RRPST session (Fig. 6F); the rat did not direct its course toward the reward sites, and spatial firing pattern was diffuse. Figure 7 shows the time course of number of rewards earned and efficiency of acquiring reward $(\mathrm{A})$, and the trajectory and firing-rate similarity (B) of this neuron across the sessions in the PLT. The rat acquired rewards gradually in the initial five sessions of the PLT, and near maximum numbers (50) of rewards in subsequent sessions. Coherent with this, traveled distance per reward rapidly decreased in the initial three sessions, and continued to gradually decrease in subsequent sessions (Fig. 7A). There were consistent changes in the trajectory similarity $\left(r_{\text {trajectory }}\right)$ and firing-rate similarity $\left(r_{\text {rate }}\right)$ (Fig. 7B), which resulted in the high correlation coefficient $(r=0.7, P<0.05)$ between these two variables. Note that both $r_{\text {trajectory }}$ and $r_{\text {rate }}$ in the RRPST following PLT learning consistently decreased as compared to those in the final PLT session. This finding reflected the fact that behavioral and spatial firing patterns in 


\section{A RRPST before PLT Learning E PLT 6th (last) Session}
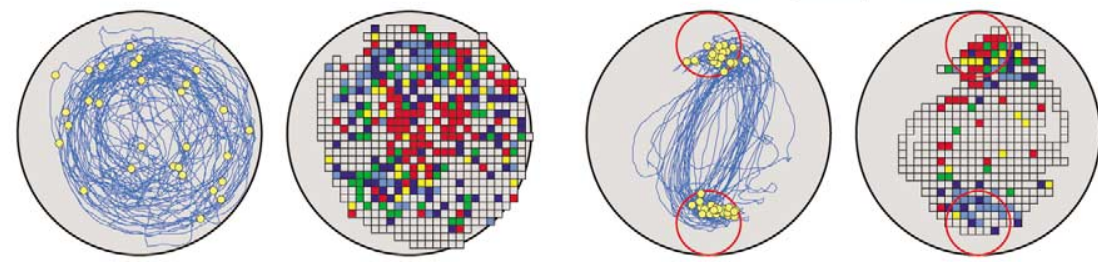

B PLT 1st Session
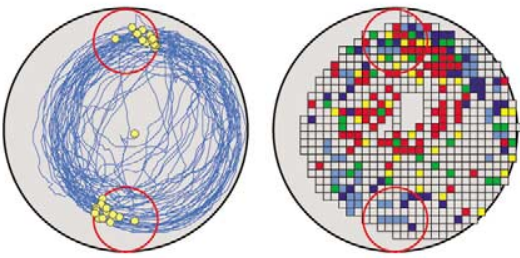

Reward at 12 o'clock Reward at 6 o'clock

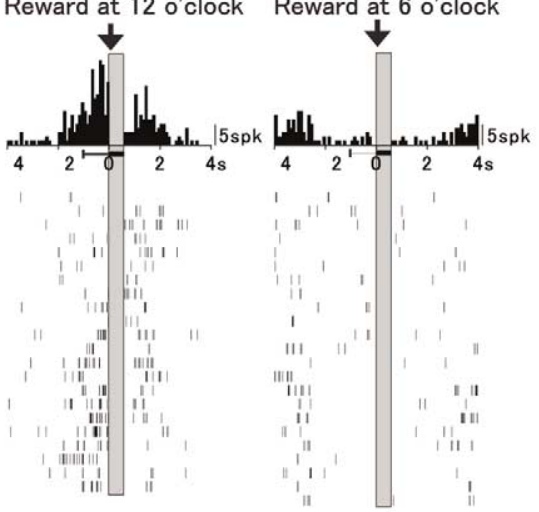

C PLT 3rd Session
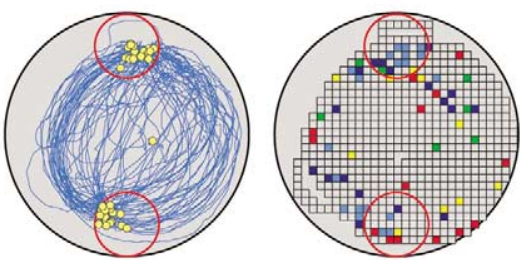

D PLT 5th Session
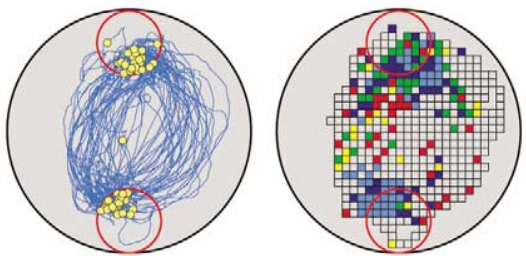

F RRPST after PLT Learning
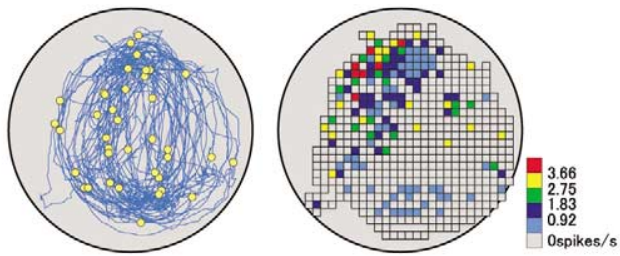

Fig. 4. An example of trajectories and firing-rate distribution of a hippocampal-formation neuron whose spatial firing pattern changed as the rat learned the place-learning task (PLT). (A) Random-reward place-search task (RRPST) before learning. (B) First session of the PLT. (C) Third session of the PLT. (D) Fifth session of the PLT. (E) Sixth and last session of the PLT. Histogram of average firing rates and raster plots in this session (below), which are time locked to onset of intracranial self-stimulation (ICSS) at reward sites at 12 or 6 o'clock positions. The gray region denotes the duration of ICSS, during which spikes were not recorded because of potential artifacts. Thin lines below abscissas, delay periods between arrival at reward site and ICSS. Thick lines below abscissas, duration of ICSS. (F) RRPST after the learning of the PLT. This neuron had no place field in the RRPST before the rat learned the PLT $(A)$ and in the first through third sessions of the PLT $(B, C)$. A place field near the reward site at the 12 o'clock position appeared in the fifth and sixth (last) session of the PLT (D, E). In the RRPST session after PLT learning, the rat persisted in shuttling between the reward sites as if they expected to find rewards at the previously learned locations. The place field established during the PLT learning was maintained (F). A-F are data from the same neuron. Other conventions as for Fig. 3.

this session did not resemble those in the last session of the PLT learning as shown in Fig. 6E and F.

\section{Relationship between trajectories and firing-rate distributions in the RRPST after PLT learning}

Of 10 complex-spike cells which changed the spatial firing patterns after PLT learning, seven neurons were tested again with the RRPST as shown in Figs. 4-7. Of these seven neurons, four $(57.1 \%)$ had reliable firing fields in the RRPST after PLT learning. To analyze the contribution of spatial firing of the seven complex-spike cells to specific trajectory patterns, similarity of trajectories $\left(r_{\text {trajectory }}\right)$ and firing-rate distributions $\left(r_{\text {rate }}\right)$ to those in the final session of the PLT was analyzed in the RRPST after PLT learning.
Figure 8 illustrates the relationship between the $r_{\text {trajectory }}$ and $r_{\text {rate }}$ of the seven neurons. Two neurons displayed high values of both $r_{\text {rate }}$ and $r_{\text {trajectory }}(r \geq 0.4)$, indicating that the behavioral and spatial firing patterns were maintained in the RRPST after PLT, while three had low values of both $r_{\text {rate }}$ and $r_{\text {trajectory }}(r<0.4)$, indicating that the behavioral and spatial firing patterns were not preserved. The analysis of variance indicated that the linear regression line fit to the data was significant $\left[F(2,4)=7.44, P<0.05 ; r^{2}\right.$ (adjusted squared multiple correlation) $=0.6]$. The $r_{\text {trajectory }}$ increased linearly when the $r_{\text {rate }}$ increased, i.e., spatial firing in the RRPST after the PLT was similar to spatial firing in the last session of the PLT when the rats displayed similar trajectories in the RRPST to those in the last ses- 

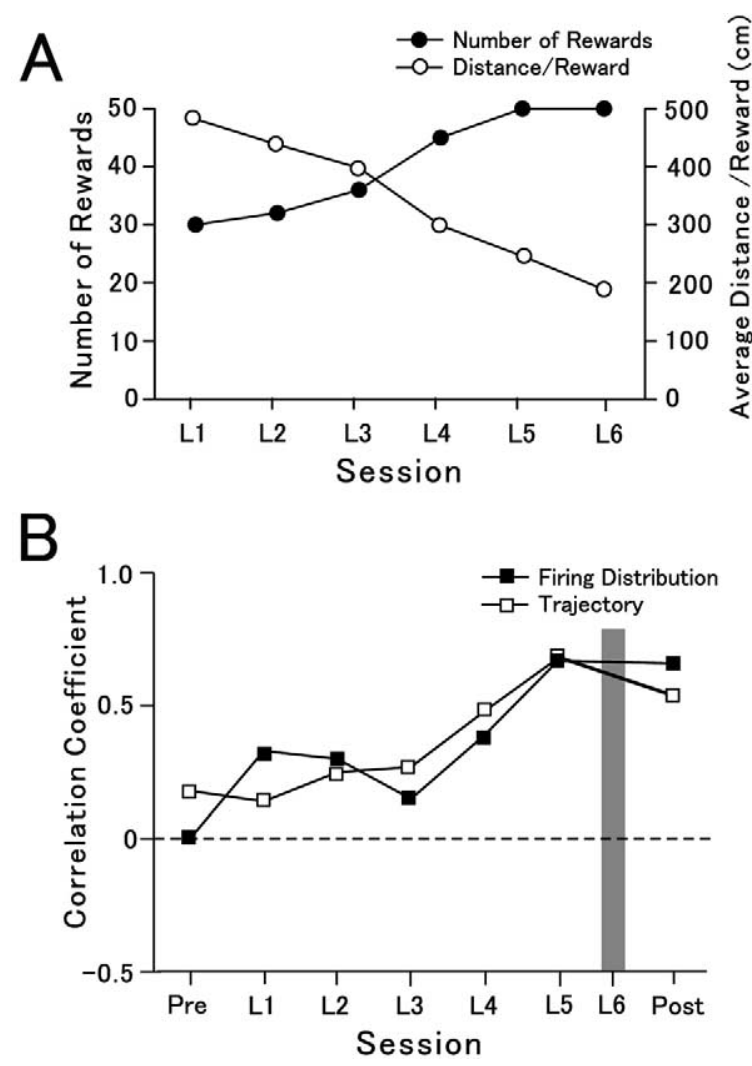

Fig. 5. Behavioral performance and changes of trajectory and firing distribution of the data shown in Fig. 4 as a function of the learning session. (A) Number of rewards and the efficiency of acquiring rewards (distance/reward) in each session. (B) Correlation coefficients of trajectory and firing distribution which were calculated between each session and the final session of the place-learning task (PLT). The gray region denotes the final session of the PLT, where both correlation coefficients were 1 . There were consistent changes in correlation coefficients of trajectory and firing distribution, which resulted in the significant correlation coefficient $(r=0.84, P<0.05)$ between these two variables, and both correlation coefficients maintained high values in the random-reward place-search task after the learning session.

sion of the PLT. This indicates causal relations between the spatial firing of the HF neurons and the movement patterns during navigation.

\section{Recording sites}

The approximate anatomical locations where the place neurons were recorded were determined histologically from small lesions made in HF after recording. Nine complex-spike cells recorded were located in the CA1 and five complex-spike cells in CA3 subfields of HF, respectively.

\section{DISCUSSION}

The present study investigated changes in the spatial firing of HF principal neurons during learning of a novel place task as measured by the efficiency of navigation strategies. We found that spatial firing of the HF neurons changed as learning proceeded. That is, changes of the HF neuron activity were well correlated to changes in rats' shuttling behaviors between reward sites. Furthermore, spatial correlates established during learning of the PLT were preserved in the RRPST after the PLT to the degree that rats maintained the same goal-directed navigation in the RRPST as appeared in the PLT.

\section{Correlates of the HF neuron activity to learning}

Previous studies have suggested at least two distinct functions of the HF in spatial behaviors, identification of locations in an environment and planning of trajectories to goals (Morris et al., 1990; Bannerman et al., 1995; Whishaw et al., 1995; Whishaw and Jarrard, 1996; Hollup et al., 2001a). The present results showed that the rats became capable of acquiring maximal numbers of rewards at a fixed location in the initial few sessions of the PLT, and, in contrast, efficiency in acquiring rewards more slowly increased throughout all sessions of it. In the last session, the rats displayed the shortest route and shuttled between the two reward sites. Recent studies in our laboratory showed that HF lesions due to transient ischemia significantly delayed learning of this PLT (Zhong et al., 2000; Hori et al., 2002). Rats with HF lesions displayed circuitous routes along a wall to reach reward sites and could not acquire rewards as efficiently as sham-operated rats. Behavioral observations in the present study along with those of these previous studies support the idea that rats can adequately learn reward-place associations in an initial stage of PLT learning, but, on the other hand, learning of efficient routes to acquire rewards develops more slowly and requires the intact HF. In the present study, the HF neuron activity changed as the latter type of learning proceeded. Therefore, it is suggested that plastic changes of HF neuronal activity contribute to the formation of efficient navigation strategies to goals rather than learning of placereward associations.

Previous experiments reported that place-cell activity has plasticity which develops in an experience-dependent manner (Bostock et al., 1991; Wilson and McNaughton, 1993; Mehta et al., 1997), and the plasticity of the place cell responses depends on NMDA receptors (Kentros et al., 1998). Extensive studies have shown that long-term potentiation, a leading candidate as a cellular mechanism for mnemonic processes (Bliss and Collingridge, 1993), requires activation of NMDA receptors, and their blockade impairs performance of spatial memory tasks (Morris and Frey, 1997; Cain, 1997). Recently, Mehta et al. (2000) showed that repetition of unidirectional trajectories through the place field changed its shape, and they suggested that this experience-dependent modulation depends on NMDA receptors. Further studies are required to investigate whether the plasticity of the HF firing shown in the present experiment is also NMDA-dependent.

\section{Role of the HF neuron activity in navigation}

Previous studies as well as the present one have reported that an alteration of reward-place contingencies leads to shifts in the location of the place fields of some HF neurons (Breese et al., 1989; Markus et al., 1995; Kobayashi et al., 1997; present results). However, Speakman and O'Keefe (1990) showed that majority of place fields were unaltered 

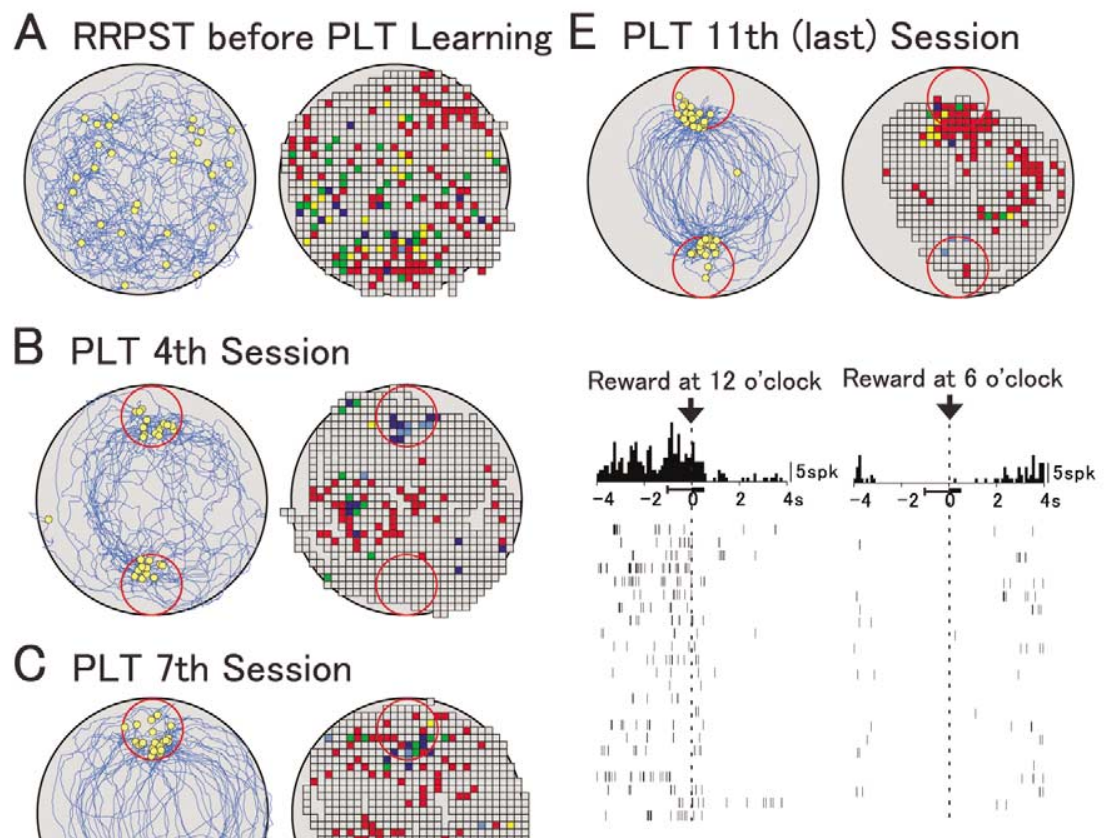

C PLT 7th Session

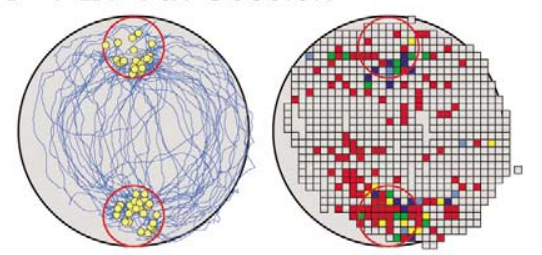

\section{F RRPST after PLT Learning}
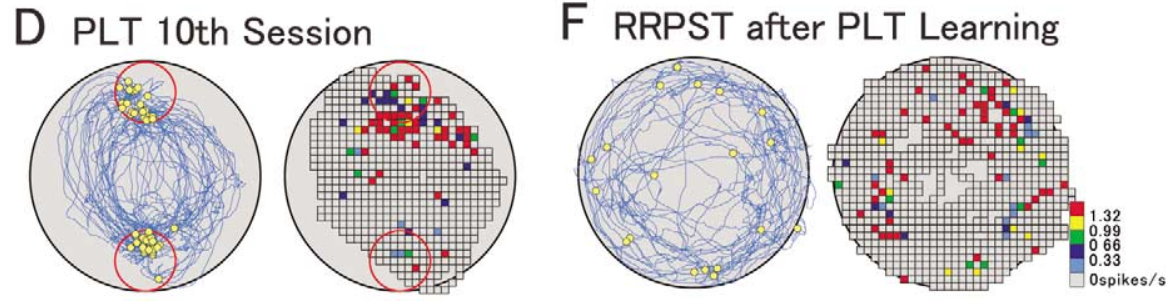

Fig. 6. Another example of trajectories and firing-rate distribution of a place cell whose spatial firing pattern changed as the rat learned the PLT. (A) Random-reward place-search task (RRPST) before learning. (B) Fourth session of the place-learning task (PLT). (C) Seventh session of the PLT. (D) Tenth session of the PLT. (E) Eleventh and last session of the PLT. The dashed line denotes the onset of intracranial self-stimulation. (F) RRPST after the learning of the PLT. This neuron had no place field in the RRPST before the rat learned the PLT (A). In seventh session of the PLT, a weak place field near the reward site at 6 o'clock appeared (C). In tenth and eleventh (last) session, the neuron showed a place field near the reward site at 12 o'clock (D, E). In the RRPST after the learning, the rat navigated randomly in the open field and the place field established during the learning was degraded $(F)$. A-F are data from the same neuron. Other conventions as for Fig. 4.

after relocation of a reward site. Speakman and O'Keefe (1990) tested rats on a plus-shaped maze, and thus, behaviors of the rats were restricted to straight movements on the arms and turns on the central platform. In the plus-shaped maze, it might not be necessary for rats to compute a precise route to a goal, and information concerning the goal location might require only the selection of an arm from the central platform. By contrast, in an openfield experiment, rats must plan a more precise route from any given position to a goal, and correct it along the trajectory if wrong directions are selected on the way (Breese et al., 1989; Markus et al., 1995; Kobayashi et al., 1997). Thus, it is likely that spatial information processing for navigation to a goal is more important in an open field than a plus-shaped maze. Previous studies have also shown that the place cell activity is influenced by the structure of the maze where the rats are navigating (McNaughton et al., 1983; Muller et al., 1994). Furthermore, some HF neurons discharge when rats approach goals
(Eichenbaum et al., 1987; Gothard et al., 1996; Hollup et al., 2001b). Such HF neuronal responses might be closely related to navigation to a goal in an open field rather than simply to passive representation of a spatial environment.

\section{Role of reward contingency in goal-directed navigation}

The present study shows that some HF neurons shift their spatial correlates along with establishment of efficient shuttling. These HF neurons robustly fired as the animal arrived at only one of the two reward sites, and continued firing during the 1-s delay before reward delivery, and decreased rapidly after the delivery. Our previous study reported comparable characteristics of place cells in the same PLT (Kobayashi et al., 1997). If the HF neurons encoded only reward contingency or reward approach behavior per se, increased firing should be observed at both reward sites. However, robust firings were observed at 


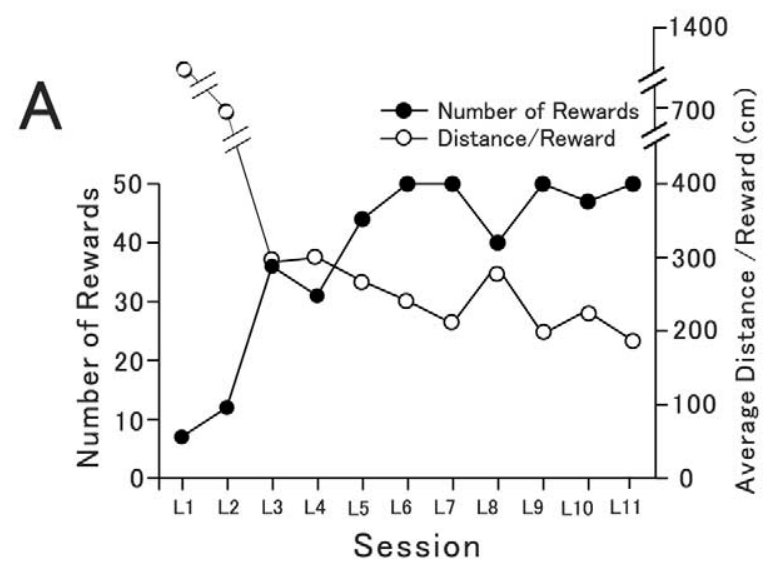

B

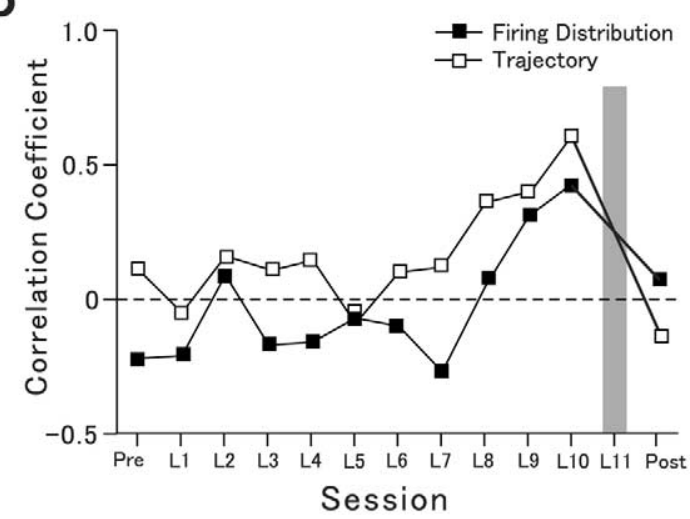

Fig. 7. Behavioral performance and changes of trajectory and firing distribution of the data shown in Fig. 6 as a function of the learning session. (A) Number of rewards per session and the efficiency of acquiring rewards (average distance/reward) in each session. (B) Correlation coefficients of trajectory and firing distribution which were calculated between each session and the final session of the placelearning task (PLT). The gray region denotes the final session of the PLT, where both correlation coefficients were 1 . There were consistent changes in correlation coefficients of trajectory and firing distribution, which resulted in the significant correlation coefficient $(r=0.7, P<0.05)$ between these two variables, and both correlation coefficients decreased in the random reward place-search task after the learning session.

only one of the two reward sites. These results suggest that the HF neuronal activity is related to reward contingency in a particular place.

In the RRPST following PLT learning, spatial firings were preserved only if the rats displayed PLT-like navigation behaviors, as if they expected to find rewards at the previously valid locations. However, the spatial firing was altered from this pattern in rats which randomly navigated at this time. These results are consistent with previous reports that spatial correlates of HF neurons were dependent on the structure of the task (Eichenbaum et al., 1987; Wiener et al., 1989; Markus et al., 1995). The task dependency of place-cell activity in the present study could be interpreted as directional dependency of the place-cell activity (McNaughton et al., 1983; Wiener et al., 1989; Muller et al., 1994; Kobayashi et al., 1997), because rats typically passed through the place field in a specific direc-

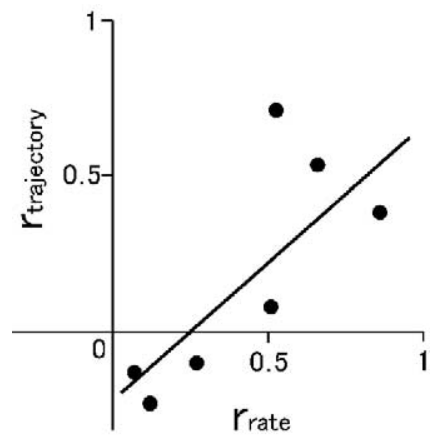

Fig. 8. Trajectory similarity versus firing-rate distribution similarity in the random-reward place-search task (RRPST) after place-learning task (PLT) learning. Similarity of trajectories $\left(r_{\text {trajectory }}\right)$ and firing-rate distributions $\left(r_{\text {rate }}\right)$ in the RRPST after PLT learning were calculated to those in the final session of the PLT, and relationship between these variables was plotted. There was a significant positive correlation between the $r_{\text {trajectory }}$ and $r_{\text {rate }}$ in the simple linear regression analysis $(P<0.05)$.

tion in the PLT but not in the RRPST. However, task dependency of the HF neuronal activity in the present task is not restricted to movement-correlated activity. In similar tasks HF neurons continued to fire until the rat acquired rewards if delays were imposed prior to reward delivery (Kobayashi et al., 1997). Furthermore, some HF neurons showed spatial firing near the reward sites in the present experiment, consistent with previous studies (Breese et al., 1989; Hollup et al., 2001b). These results suggest that activity of some HF neurons reflects critical variables required for task solving such as structuring behavior to deal with varying reward contingency or an attitude to a given situation where reward is available after specific behaviors.

\section{Theoretical consideration of HF involvement in navigation}

The present experiment showed that spatial firing of HF neurons changed in correlation with learning of the PLT. Firing patterns of the HF neurons represent the environment, that is a cognitive map (O'Keefe and Nadel, 1978). In this view, the present results could be interpreted as creation of a novel representation of the environment. An alternative interpretation to this account, however, is that the firing changes were attributed to changes in trajectories which were acquired during learning. A recent theory of hippocampal representation by Eichenbaum et al. (1999) suggests that coding of spatial locations by HF place cells emerges from a fundamental representation of behavioral episodes, leading to the idea that spatial firing of place cells reflects a specific action of the rat at a specific location. The present experiment supports the second interpretation, because changes of spatial firing established during learning disappeared if the rat did not take the routes which had been acquired.

A direct route to a goal can be computed by taking the difference between a vector which describes the rat's current positional orientation and a vector which gives the position of the goal (Collett et al., 1986; McNaughton et al., 1994), and these vectors are encoded by the place-cell 
activity because spatial firing patterns of the place cell represent a certain distance and an allocentric bearing from a landmark (McNaughton et al., 1994). Subtraction of a vector representing a current position from a vector encoding a reward site might provide a direct route to a goal, and then rats could shuttle efficiently between the reward sites. Recently, Frank et al. (2000) demonstrated that the neuron in the HF and entorhinal cortex differentially fired at a given location depending on the past or future path of rats. Furthermore, these characteristics were more evident in the HF as well as the deep layers of the entorhinal cortex, which receives the primary outputs of the HF (Witter et al., 1989), than its superficial layers. These results suggest that planning of an entire route from a starting point to a goal is computed within the HF, and the output of the computation could be provided through the deep layers of the entorhinal cortex to other related structures. Vector subtraction between a start point and a goal might be performed within the HF.

\section{CONCLUSIONS}

The present study showed that plasticity of the HF neuron activity contributed to the formation of efficient navigation strategies to goals, and suggests that the activity of HF neurons might not merely serve to represent the current position of animals, but be associated with variables which have a major behavioral significance to solve a task. The HF neuron activity might represent the manner how animals have a significant relation to an environment as well as physical structures which consist of the environment.

Acknowledgements-This work was supported by Japanese Ministry of Education, Culture, Sports, Science and Technology Grant-in-Aid for Scientific Research (11308033, 12210009, and 12680792). We thank Dr. Sidney I. Wiener, CNRS-College de France, for suggestions on a draft of the manuscript.

\section{REFERENCES}

Abrahams S, Pickering A, Polkey CE, Morris RG (1997) Spatial memory deficits in patients with unilateral damage to the right hippocampal formation. Neuropsychologia 35:11-24.

Bannerman DM, Good MA, Butcher SP, Ramsay M, Morris RG (1995) Distinct components of spatial learning revealed by prior training and NMDA receptor blockade. Nature 378:182-186.

Bliss TV, Collingridge GL (1993) A synaptic model of memory, longterm potentiation in the hippocampus. Nature 361:31-39.

Bostock E, Muller RU, Kubie JL (1991) Experience-dependent modifications of hippocampal place cell firing. Hippocampus 1:193-205.

Breese CR, Hampson RE, Deadwyler SA (1989) Hippocampal place cells: stereotypy and plasticity. J Neurosci 9:1097-1111.

Bures J, Fenton AA, Kaminsky Y, Rossier J, Sacchetti B, Zinyuk L (1997a) Dissociation of exteroceptive and idiothetic orientation cues: effect on hippocampal place cells and place navigation. Philos Trans R Soc Lond B Biol Sci 352:1515-1524.

Bures J, Fenton AA, Kaminsky Y, Zinyuk L (1997b) Place cells and place navigation. Proc Natl Acad Sci USA 94:343-350.

Cain DP (1997) LTP, NMDA, genes and learning. Curr Opin Neurobiol $7: 235-242$.

Collett TS, Cartwright BA, Smith BA (1986) Landmark learning and visuo-spatial memories in gerbils. J Comp Physiol 158:835-851.

Eichenbaum H, Dudchenko P, Wood E, Shapiro M, Tanila H (1999)
The hippocampus, memory, and place cells: is it spatial memory or a memory space? Neuron 23:209-226.

Eichenbaum H, Kuperstein M, Fagan A, Nagode J (1987) Cue-sampling and goal-approach correlates of hippocampal unit activity in rats performing an odor-discrimination task. J Neurosci 7:716-732.

Eichenbaum H, Mathews P, Cohen NJ (1989) Further studies of hippocampal representation during odor discrimination learning. Behav Neurosci 1037:1207-1216.

Frank LM, Brown EN, Wilson M (2000) Trajectory encoding in the hippocampus and entorhinal cortex. Neuron 27:169-178.

Gothard KM, Skaggs WE, Moore KM, McNaughton BL (1996) Binding of hippocampal CA1 neural activity to multiple reference frames in a landmark-based navigation task. J Neurosci 15:823-835.

Hollup SA, Kjelstrup KG, Hoff J, Moser MB, Moser El (2001a) Impaired recognition of the goal location during spatial navigation in rats with hippocampal lesions. J Neurosci 15:4505-4513.

Hollup SA, Molden S, Donnett JG, Moser MB, Moser EI (2001b) Accumulation of hippocampal place fields at the goal location in an annular watermaze task. J Neurosci 21:1635-1644.

Hori E, Uwano T, Tamura R, Miyake N, Nishijo H, Ono T (2002) Effects of a novel arginine-vasopressin derivative, NC-1900, on the spatial memory impairment of rats with transient forebrain ischemia. Cognitive Brain Res 13:1-15.

Jarrard LE (1993) On the role of the hippocampus in learning and memory in the rat. Behav Neural Biol 60:9-26.

Kentros C, Hargreaves E, Hawkins RD, Kandel ER, Shapiro M, Muller RV (1998) Abolition of long-term stability of new hippocampal place cell maps by NMDA receptor blockade. Science 280:2121-2126.

Kobayashi T, Fukuda M, Ono T, Eifuku S (1991) Plasticity of rat hippocampal place cell responsiveness relative location of rewarding ICSS delivery. Soc Neurosci Abstr 17:483.

Kobayashi T, Nishijo H, Fukuda M, Bures J, Ono T (1997) Taskdependent representations in rat hippocampal place neurons. J Neurophysiol 78:597-613.

Lenck-Santini PP, Save E, Poucet B (2001) Evidence for a relationship between place-cell spatial firing and spatial memory performance. Hippocampus 11:377-390.

Maguire EA, Burke T, Phillips J, Staunton H (1996) Topographical disorientation following unilateral temporal lobe lesions in humans. Neuropsychologia 34:993-1001.

Maguire EA, Frackowiak RS, Frith CD (1997) Recalling routes around London: activation of the right hippocampus in taxi drivers. J Neurosci 17:7103-7110.

Maguire EA, Burgess N, Donnett JG, Frackowiak RS, Frith CD, O'Keefe J (1998) Knowing where and getting there: a human navigation network. Science 280:921-924.

Markus EJ, Qin YL, Leonard B, Skaggs WE, McNaughton BL, Barnes CA (1995) Interactions between location and task affect the spatial and directional firing of hippocampal neurons. J Neurosci 15:70797094.

Matsumura N, Nishijo H, Tamura R, Eifuku S, Endo S, Ono T (1999) Spatial- and task-dependent neuronal responses during real and virtual translocation in the monkey hippocampal formation. $\mathrm{J}$ Neurosci 19:2381-2393.

McHugh TJ, Blum KI, Tsien JZ, Tonegawa S, Wilson MA (1996) Impaired hippocampal representation of space in CA1-specific NMDAR1 knockout mice. Cell 87:1339-1349.

McNaughton BL, Barnes CA, Gerrard JL, Gothard K, Jung MW, Knierim JJ, Kudrimoti H, Qin Y, Skaggs WE, Suster M, Weaver KL (1996) Deciphering the hippocampal polyglot: the hippocampus as a path integration system. J Exp Biol 199:173-185.

McNaughton BL, Barnes CA, O'Keefe J (1983) The contributions of position, direction, and velocity to single unit activity in the hippocampus of freely-moving rats. Exp Brain Res 52:41-49.

McNaughton BL, Barnes CA, Meltzer J, Sutherland RJ (1989) Hippocampal granule cells are necessary for normal spatial learning but not for spatially-selective pyramidal cell discharge. Exp Brain Res 76:485-496. 
McNaughton BL, Knierim JJ, Wilson MA (1994) Vector encoding and the vestibular foundations of spatial cognition, a neurophysiological and computational mechanisms. In: The cognitive nerosciences (Gazzaniga M, ed), pp 585-595. Boston: MIT Press.

Mehta MR, Barnes CA, McNaughton BL (1997) Experience-dependent, asymmetric expansion of hippocampal place fields. Proc Natl Acad Sci USA 94:8918-8921.

Mehta MR, Quirk MC, Wilson MA (2000) Experience-dependent asymmetric shape of hippocampal receptive fields. Neuron 25:707-715.

Mellet E, Briscogne S, Tzourio-Mazoyer N, Ghaem O, Petit L, Zago L, Etard O, Berthoz A, Mazoyer B, Denis M (2000) Neural correlates of topographic mental exploration, the impact of route versus survey perspective learning. Neuroimage 12:588-600.

Morris RG, Frey U (1997) Hippocampal synaptic plasticity: role in spatial learning or the automatic recording of attended experience? Philos Trans R Soc Lond B Biol Sci 352:1489-1503.

Morris RGM, Schenk F, Tweedie F, Jarrard LE (1990) Ibotenate lesions of hippocampus and/or subiculum, dissociating components of allocentric spatial leaning. Eur J Neurosci 2:1016-1028.

Muller RU, Bostock E, Taube JS, Kubie JL (1994) On the directional firing properties of hippocampal place cells. J Neurosci 14:72357251.

Muller RU, Kubie JL (1987) The effects of changes in the environment on the spatial firing of hippocampal complex-spike cells. J Neurosci 7:1951-1968.

Muller RU, Kubie JL, Ranck JB Jr (1987) Spatial firing patterns of hippocampal complex-spike cells in a fixed environment. J Neurosci 7:1935-1950.

Nishijo H, Kobayashi T, Tamura R, Matsumoto G, Ono T (1999) Reward anticipation-related neural activity in the rat hippocampus during spatial navigation. Soc Neurosci Abstr 25:1382.

Nishijo H, Kita T, Tamura R, Eifuku S, Terasawa K, Ono T (1997) Motivation-related neuronal activity in the object discrimination task in monkey septal nuclei. Hippocampus 7:536-548.

O'Keefe J, Burgess N (1996) Geometric determinants of the place fields of hippocampal neurons. Nature 381:425-428.

O'Keefe J, Conway DH (1978) Hippocampal place units in the freely moving rat: why they fire where they fire. Exp Brain Res 31:573590.

O'Keefe J, Dostrovsky J (1971) The hippocampus as a spatial map: preliminary evidence from unit activity in the freely moving rat. Brain Res 34:171-175.

O'Keefe J, Nadel L (1978) The hippocampus as a cognitive map. Clarendon: Oxford.

O'Keefe J, Speakman A (1987) Single unit activity in the rat hippocampus during a spatial memory task. Exp Brain Res 68:1-27.

Olton DS, Becker JT, Handelmann GE (1979) Hippocampus, space, and memory. Behav Brain Sci 2:313-365.

Ono T, Nakamura K, Fukuda M, Tamura R (1991) Place recognition responses of neurons in monkey hippocampus. Neurosci Lett 121 194-198.

Ono T, Nakamura K, Nishijo H, Eifuku S (1993) Monkey hippocampal neuron related to spatial and nonspatial functions. J Neurophysio 70:1516-1529.

Paxinos G, Watson C (1986) The rat brain in stereotaxic coordinations, 2nd. Academic Press: San Diego.

Sakurai Y (1996) Hippocampal and neocortical cell assemblies encode memory processes for different types of stimuli in the rat. J Neurosci 16:2809-2819.

Sasaki K, Ono T, Nishino H, Fukuda M, Muramoto K (1983) A method for long-term artifact-free recording of single unit activity in freely moving, eating and drinking animals. J Neurosci Methods 7:43-47.

Skaggs WE, McNaughton BL (1998) Spatial firing properties of hippocampal CA1 populations in an environment containing two visually identical regions. J Neurosci 18:8455-8466.

Speakman A, O'Keefe J (1990) Hippocampal complex spike cells do not change their place fields if the goal is moved within a cue controlled environment. Eur J Neurosci 2:544-555.

Sharp PE, Kubie JL, Muller RU (1990) Firing properties of hippocampal neurons in a visually symmetrical environment: contributions of multiple sensory cues and mnemonic processes. J Neurosci 10: 3093-3105.

Thompson LT, Best PJ (1990) Long-term stability of the place-field activity of single units recorded from the dorsal hippocampus of freely behaving rats. Brain Res 509:299-308.

Whishaw IQ, Cassel JC, Jarrad LE (1995) Rats with fimbria-fornix lesions display a place response in a swimming pool: dissociation between getting there and knowing where. J Neurosci 15:57795788.

Whishaw IQ, Jarrard LE (1996) Evidence for extrahippocampal involvement in place learning and hippocampal involvement in path integration. Hippocampus 6:513-524.

Wiener SI, Paul CA, Eichenbaum H (1989) Spatial and behavioral correlates of hippocampal neuronal activity. J Neurosci 9:27372763.

Wilson MA, McNaughton BL (1993) Dynamics of the hippocampal ensemble code for space. Science 261:1055-1058.

Witter MP, Groenewegen HJ, Lopes da Silva FH, Lohman AH (1989) Functional organization of the extrinsic and intrinsic circuitry of the parahippocampal region. Prog Neurobiol 33:161-253.

Zhong YM, Nishijo H, Uwano T, Tamura R, Kawanishi K, Ono T (2000) Red ginseng ameliorated place navigation deficits in young rats with hippocampal lesions and aged rats. Physiol Behav 69:511525.

Zinyuk L, Kubik S, Kaminsky Y, Fenton AA, Bures J (2000) Understanding hippocampal activity by using purposeful behavior: place navigation induces place cell discharge in both task-relevant and task-irrelevant spatial reference frames. Proc Natl Acad Sci USA 97:3771-3776. 